\title{
SOUNDTRAD, A METHOD AND TOOL FOR PROTOTYPING AUDITORY DISPLAYS: CAN WE APPLY IT TO AN AUTONOMOUS DRIVING SCENARIO?
}

\author{
Doon MacDonald \\ CHERISH-DE \\ The Cherish Digital Economy Centre \\ Swansea University, UK \\ d.g.macdonaldeswansea.ac.uk
}

\author{
Tony Stockman \\ Queen Mary University of London \\ Centre for Digital Music \\ London, UK \\ t.stockman@qmul.ac.uk
}

\begin{abstract}
This paper presents SoundTrAD, a method and tool for designing auditory displays for the user interface. SoundTrAD brings together ideas from user interface design and soundtrack composition and supports novice auditory display designers in building an auditory user interface. The paper argues for the need for such a method before going on to describe the fundamental structure of the method and construction of the supporting tools. The second half of the paper applies SoundTrAD to an autonomous driving scenario and demonstrates its use in prototyping ADs for a wide range of scenarios.
\end{abstract}

\section{INTRODUCTION}

In this paper we describe a method and toolkit for prototyping auditory displays (ADs). We argue that there is a lack of methods for supporting novice designers who want to design ADs, specifically a lack of methods that enable early-stage prototyping of an $\mathrm{AD}$ design. The method we describe, called SoundTrAD, takes, as its basis, a conceptual blending [1] of techniques from soundtrack composition and from user interface design, to form a novel and playful approach to AD design for the human-computer interface. In this paper we describe its current application and future potential rather than going into specific detail of how SoundTrAD was developed. Interested readers can reference [2] and [3] to learn more around how SoundTrAD was conceptualised, developed and evaluated.

In this paper (section 4) we propose one hypothetical design problem and apply SoundTrAD to exemplify its use. This involves the creation of an auditory display for an autonomous vehicle scenario. We demonstrate that SoundTrAD can help novice designers think about certain design issues for ADs such as the employment of use-case scenarios, sound masking and aesthetics. We also argue that its flexible in the level of support it can offer the designer depending on their experience. We conclude by discussing ideas for further design and development of SoundTrAD. Commercial 4.0 International License. The full terms of the License are available at http://creativecommons.org/licenses/by-nc/4.0

\section{BACKGROUND}

There are many approaches to creating auditory interfaces in existance. These range from approaches that focus on the conceptual stages of the design problem [4], to those that focus more on specific mapping techniques of information to sound [5]. However, after Frauenberger, Stockman and Bourguet [6] carried out a survey and collated approaches to auditory display design, the first and second author still concluded in a later publication that, despite the number of approaches, 'unsupported design decisions were found on all levels of the design process' [7]. It has been observed that there is the lack of methods that enable the designer to move successfully from a conceptual design to a physical prototype of their interface [8] and it is arguable that this could be down to the lack of centralised design knowledge as proposed by [7]. As Brazil and Fernstrom [4] observed, there is no single method that can deal with the complex socio-cultural context of auditory display design. It is thought that the methods for creating auditory displays are ad-hoc, scattered across a number of sources and not accessible to novice interface designers $[7,8]$ and that sound design for user interfaces is often based on intuition and available technology, rather than a framework or theoretical analysis [9]. It has also been argued that methods that enable a novice designer to build an auditory display creatively are not fully developed and that design knowledge is not disseminated outside of auditory display design experts. This is exemplified by the observation from Stephen Barrass and Christopher Frauenberger that 'the knowledge of how to build a 'good' auditory display is hidden in the experience of the experts and creativity of the artists' [10]. This highlights the importance of de-mystifying the process of creating an auditory display for novice designers. There are, after all, potential benefits to considering the role of the novice designer, as it could lead to the importance of sound in user interfaces becoming more widely appreciated.

It has been argued that sounds need to be consistent across the interface and need to be tested for effectiveness and increased interface usability [11].

\section{WHAT IS SOUNDTRAD?}

SoundTrAD is motivated by the need for a method that is accessible to novice audio interface designers and furthermore, introduces the field of audio interface design to a wide group of people. SoundTrAD also caters for the need for aesthetics when designing ADs, something that Vickers [12] argues is missing. SoundTrAD is based on a blending of ideas from user interface design 
and soundtrack composition. Parallels between the two disciplines that underpin the SoundTrAD method are illustrated in Figure 1 and a summarising explanation of how this 'blend' was formed to inform the method stages and steps of SoundTrAD is documented in section 3.3. Section 3.4 then describes the tools that were designed to support the method stages and steps.

\subsection{Stages of Interface Design}

Existing guidelines for interface design are often made up of several methodological stages. The following stages are derived from a range of sources on interface development, including $[13,14,15,16,17]$ and represent a summary of interface stages accounted in those references. The different sources vary to some degree in terminology and emphasis they place on each of the stages, however, these stages provide a broad structure from which to approach the design of an interface.

\section{Requirements Gathering Events}

The first of the stages is the requirements investigation or requirements gathering stage. This stage involves identifying who the users are, the tasks to be supported by the interface and the context of use. Often at this stage a designer will identify actions and objects within the interface that relate to the human interaction [15].

\section{Conceptual Design}

The second stage, the conceptual design stage, involves considering the overall form of the interface including the modes of interaction to be supported and how communications between the users and the system are to be organised.

\section{Detailed Design Stage}

The third stage is the physical or detailed design stage whereby the interface is mapped, refined, implemented and prototyped. When discussing these stages of developing interfaces specifically for auditory displays, Peres et al. [16] point out that in 'whatever form it takes, the specification should detail how the interface will be organised, how it will sound and appear and how it will behave in response to user-input well enough to prototype or implement the auditory task to a point that is sufficient for subsequent development and evaluations' (p.173)

\section{Evaluation}

Finally, there is the evaluation stage, whereby the interface is tested with end-users/ stake holders $[17,16]$. It is usual that evaluation is done iteratively throughout the development of the interface, so the designer effectively iterates between the last 3 stages in order to better inform the design process.

\subsection{Soundtrack Composition}

The following section provides a review of the functions and principles of creating soundtracks. This review is based on multiple sources including [18, 19, 20, 21, 22, 23]. The different sources vary to some extent in the way they describe the principles and functions of soundtrack composition, but the review given here draws on what is considered to be the most frequently utilised and widely held principles. Once again, given the overall goal of the SoundTrAD method, we particularly focus on the concepts in soundtrack composition practice that could be seen to parallel practices in interface development.

\section{Spotting}

Composers will analyse a scene by 'spotting' it for places that could be enhanced by audio. Hollywood sound designer, David Sonnenschein wrote that within every on-screen character, object and action there is potential to generate a sound that can enhance the narrative and story [19]. Sonnenschein identifies the following 'voices' to listen out for in order to identify key storytelling elements that can be 'amplified by sound'; namely by identifying and circling explicit words and phrases: People, objects, actions, environments, emotions and transitions.

\section{Arranging}

Once the composer has annotated their ideas for sounds, the suggested ideas are classified into categories of dialogue, music, sound effects (D-M-Es). Additionally, the Foley track is created (whereby actions sounds are created to sync with on-screen actions). What is important in a soundtrack is the consideration of how these sounds work together and how they form the bigger soundtrack by relating to one another and to the story being told. Within a film, the soundtrack contains not only the musical score, but ambient sound, dialogue, sound effects, and silence. To underline the importance of considering the soundtrack and all the sounds that make it up as a whole, Lipscomb and Tolchinsky argued for the analysis of the entire soundtrack because 'musical sound, dialogue, sound effects, silence, and some sounds that fall in the cracks between traditional categories all exist for the purpose of enhancing the intended message of the motion picture' (p.5) [24]

\section{Composing/Sampling/Syncing}

This stage involves sourcing the sound samples and/or composing original music. The stage also involves arranging the Foley track and creating sound effects.

\section{Evaluation/Auditioning}

This is inherent in soundtrack composition as it forms a natural part of auditioning the sounds and going back to previous stages to revisit and revise arrangements and ideas.

\subsection{Similarities: the Stages of SoundTrAD}

Figure 1 presents four major stages of interface design and four major stages of soundtrack composition and steps within these stages. Figure 1 portrays existing similarities between how a film scene and an HCI scenario are analysed. Specifically, the importance that both soundtrack composition and auditory interface design place on identifying and working with actors (users) or characters as part of the spotting stage and requirements gathering stage respectively. Identifying actions, objects, locations and context is also important to both disciplines. Both disciplines, as part of the detailed design stage and composing stage, respectively, also place importance on considering suitable audio onto which events can be mapped. Finally, both disciplines depend on the ability to evaluate and iterate their respective design processes.

\section{A Blended Space}

In their recent studies on designing natural and intuitive interfaces [25] described conceptual blending as 'the ability to combine multiple conceptual spaces into one emergent one, called a 'blend'. 


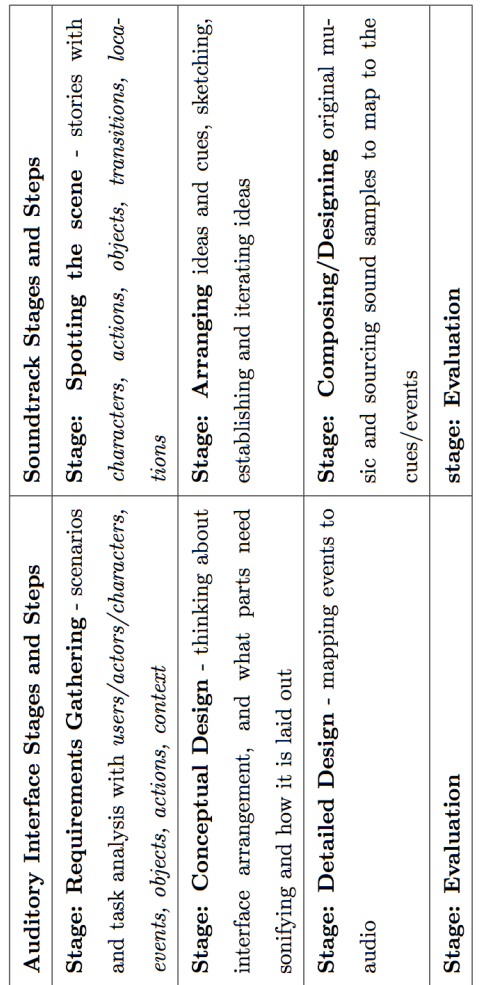

Parallels Between The Two Disciplines

Figure 1: Parallels Between Soundtrack Composition and Interface Design

Fauconnier and Turner also argued that 'a conceptual blend operates in two input mental spaces to yield a third space, the blend' and that 'partial structure from the input spaces is projected into the blended space, which has an emergent structure of its own.' [1].

Figure 2 presents an initial bringing together of these stages and steps based on the parallels identified. This represents a candidate blended space between the two disciplines and formed the basis of SoundTrAD. The principle method stages are consistent with the stages used for interface design: requirements gathering, conceptual design, detailed design and evaluation. This supports the goal of using SoundTrAD to develop auditory interfaces. The finer steps within these stages are synthesised from both disciplines of interface design and soundtrack composition.

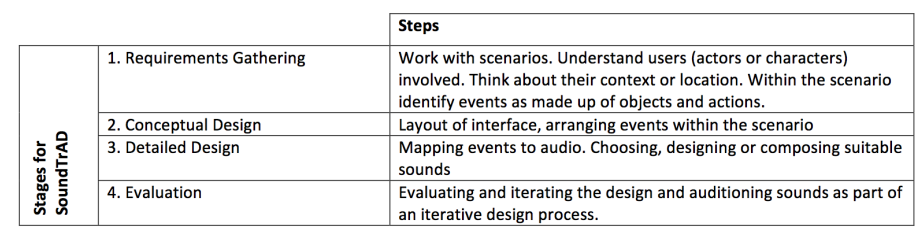

A Blended Space

Figure 2: Framework for SoundTrAD-method stages and steps

\subsection{The Tools}

There are, to-date, a set of prototype tools that were developed along side the method concept. They are based on techniques employed in soundtrack composition and were designed to support important aspects of the method and act as a proof of concept.

\subsubsection{Cue Sheet}

The cue sheet (Figure 3) helps the designer/user document the information that needs to be sonified within the scenario. Specifically, the events, their timings and their types. This makes the cue sheet a good document to refer back to at a later date. It is particularly useful if the events are being moved around (see timeline) as part of the arranging stages of the method and the designer wants to see the original layout, justification for design, thoughts etcetera or wants to share ideas with other sound designers and capture design rationale. The columns on the cue sheet, from left to right are labelled as follows:

1. Time.

2. Description /Value.

3. Cause and Associated Events.

4. Number of events to be sonified.

The cue sheet is filled out from top to bottom whereby each requirement is mapped out on its own horizontal line. The user can enter whatever they like into the columns and rows, however, some guidelines are offered when it comes to filling out the column labelled 'cause and associated events'.

Events: The current version of SoundTrAD identifies the following causes and associated events that the user can select from. The idea is that they will map to suggestions in the database of sound ideas. The causes that are listed below initially are based on event descriptions in research by [26].

The causes are as follows:

- Start

- Stop

- State Changes

- Threshold

The events are as follows:

- User Actions (UA): a user triggers an event

- System Actions/Events (SA): a system triggers an event

- Environmental Events (EE): natural occurrences are classed as events

- Continuous Events (CE): an event that lasts all or most of the duration of the use case scenario

The aim of the cue sheet is to capture design rationale and to gather both qualitative and quantitative data about each requirement before they are mapped to suggested sounds.

\subsubsection{The Database}

After the cue sheet is filled out with the requirements, the designer can access a database of sound suggestions.

When relating auditory display to soundtrack composition, then its important to think about how the sounds blend and work 


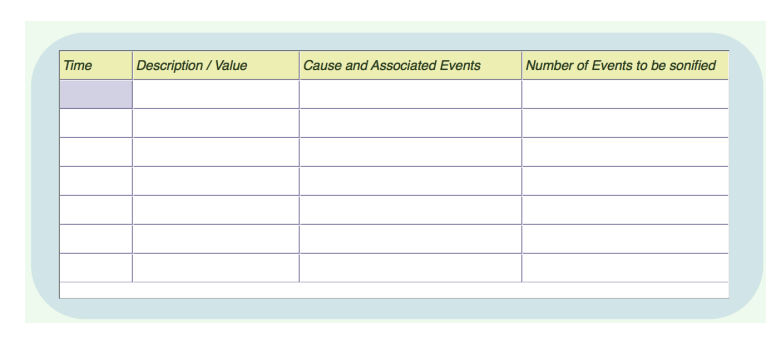

Empty Cuesheet

Figure 3: An Example Cue sheet

together to help communicate what ever is they are meant to communicate within the scenario. The stages and steps of SoundTrAD (the blended space, outlined above) should support this attention to detail with the mapping whilst a larger method structure is kept in place. In turn this helps the designer in the design process (not lose track of design thinking, ideas, iterations etcetera whilst focussing on sound and mapping).

All the different 'types' of sound that make up a soundtrack are catered for and represented in a database of sound samples: music, background, Foley, sound effects and ambient soundscapes.

Additionally, familiar soundtrack composition techniques are utilised such as the use of concrete sounds to represent actions, motifs to help thematic development and and drones to enhance a sense of space and a sense of time passing. Importantly all the different types of sounds can be constructed and layered to build a soundtrack that users can customise when mapping.

A set of guidelines for adding sounds to the interface were devised by Hildebrandt and Rinderle-Ma [26]. These are used as guidelines within SoundTrAD they were included to cater for different types of sounds and are particularly aligned to guidelines for soundtrack composition. They are cited below:

1. 'Users should be able to customise the mapping from data to sound'.

2. 'If concrete auditory representations for the occurring events are available, the use of Auditory Icons can produce positive results'.

3. 'When occurring events are mapped to Earcons, complex timbres (possibly based on real-world instruments) should be preferred over simple timbres (like sine waves)'.

4. 'The Earcons should take concepts from the areas of motif design and melodic contours into consideration and adhere to "musical" concepts (such as the western tonal system)'.

5. 'If motifs are being applied, they should differ not only in pitch but also in rhythm and intensity'.

6. 'Different concepts can be conveyed by using different motifs (possibly hierarchically structured) and/or different timbres'.

7. 'In general, rhythm and percussion should be included in sonifications'.

8. 'Continuous sounds (such as drones) should be used to convey the duration of ongoing activities'.

For the first investigation of the method and tools described here, we used a database of 80 sounds. The sounds were based on the guidelines quotes above and were categorised as follows:

\section{- Sound Effects/Foley}

- Ambience, environmental and background drones

- One off chimes, 'dings' and beeps within set scales (minor, major)

- A set of motifs based on these scales.

For the investigation of a specific case research should be undertaken to investigate whether there is empirically evidence on which to base the choice of sounds, this should be particularly the case where safety is involved, or the system is is critical or unstable state. It should, be remembered at this stage the system is providing a prototyping platform and again, in the case of safety critical system will be subjected to exhaustive simulation.

\subsubsection{The Timeline}

The timeline, as shown in Figure 4, caters for the ability to audition sounds, re-document ideas and move events around within a given use-case to cater for non-linear interactions in time. The events are represented from left to right and from top to bottom. Time is represented from left to right which means that events that occur at the same time will appear vertically in line and will sound together. Specifically, the timeline supports the modelling of different event sequences and combinations and repeated interactions.

This gives the designer the chance to check for issues with aesthetics and masking. The events can be moved using the mouse and the start and stop buttons can control the playback and moving grid line.

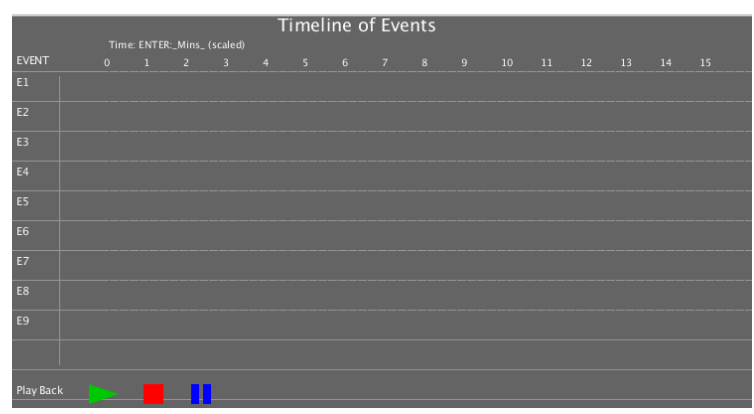

Blank Timeline

Figure 4: The Timeline. Events have not been filled in yet. The number of event (E) lines, represented here on the vertical axis) can be added to and taken away. The time can be shortened and lengthened and changed to a suitable scale to cater for the designer's needs

\subsection{Latest Findings and Potential Applications}

An evaluation of SoundTrAD with the steps and tools described above is described in [3]. 11 participants took part in the study and were mostly new to $\mathrm{AD}$ design.

At the end of the study we asked the participants to write down any scenarios to which they felt they could apply SoundTrAD. The responses demonstrate the wide-range of scenarios and applications that participants felt the system could be used for. Listed below are accounts of these participant $(\mathrm{P})$ testimonials as potential application areas for SoundTrAD at this stage of its development. 
P1: "Sport events, data sonification, live data performance tool".

P2: "I would like if it can respond to real time events. Then it can be used in public areas such as cafeterias. The system can be related to the play list the cafe is using. When there are more customers in the shop, play some pop music or music with a quick tempo, customer might eat faster and leave quicker. When there are only few people in the cafe, play some classic music to ease the pressure of customers."

P3: "Analysis of any complex system e.g. scientific research"

P4: "For tasks or cases that need constant monitoring and need to fire alerts if changes happen, while users are occupied doing something else. Almost like a background monitor."

P5: "Petrol consumption. When client payments made to bank account (might be too complex but would be really helpful) Metered water consumption. When I need coffee!"

P6: "I think it can be applied to use with any storylinelevents that needs auditory display"

P7: "Music education, aiding system for sensory-impaired people, mobile application, animal monitoring, and so on."

P8: "Transport hubs like railways stations and airports, either for people (queues building up, need more staff on check in desks etc) or for the logistics of moving bags (airports) or rolling stock (sending in more carriages) or even having an alert for a problem with trains and getting rail replacement busses."

P9: "Digital archives. Revision aids. Teaching aids especially for students with special educational needs'. I would really like to use with historical data being archived online. I think this kind of audio display would be great for a variety of students needs and could make education more accessible for all. There are endless possibilities here I think'

P10: 'To help workers 'keep an ear' on their work. To help supervisors monitor staff activity. To help managers review data and plan accordingly. To add auditory and notification to already existing IT systems (in vehicles/computers/classroom environments). Hugely beneficial to people with impairments."

P11: "in vehicles for optimum performance and monitoring. For use in medical and sporting monitoring equipment. for monitoring energy use in the home/business. for use in extreme locations for monitoring yourself/the environment. to aid a person with disability/medical condition. For anyone that is multitasking any situation and needs to monitor and respond to situations while engaged in another activity"

\section{APPLYING SOUNDTRAD TO A DRIVING SCENARIO}

In this paper we apply SoundTrAD to the design of an in-vehicle $\mathrm{AD}$. We feel that a driving scenario is very suitable to exemplify the potential of SoundTrAD, particularly in regards to demonstrating how soundtrack composition can influence the design of the auditory display.

For example, a driver will often maintain two or more tasks whilst driving, such as navigating the road whilst listening to music and paying attention to collision warning sounds or sounds that indicate a seat-belt is not done up, or the fuel is low. Additionally, it is arguable that the natural sounds from the road and car engine also play an important part when it comes to successful and safe driving.

This scenario therefore already has a potential construct of Fo- ley sounds, environmental/location/ contextual sounds and music and possibly speech all vying for bandwidth. Consequently, there will be issues with masking, aesthetics and acceptability of the interface [11].

A soundtrack is normally made up of these elements and designers, if using SoundTrAD, will 'spot' the scene to map out sounds and then use a cue sheet. Therefore, it is felt that SoundTrAD (as a method and tool that takes ideas from soundtrack composition) gives the designer a better chance of addressing these issues when designing the auditory display for this scenario.

\subsection{The steps and the Tool (applied):}

\subsubsection{Stage 1: A Scenario}

1. The Scenario: Autonomous Motorway Driving

2. The User: A solo driver

3. Things you Want to Find Out: When as a driver you need to pay attention to the auditory display, giving you the option to be aware of choices the car is making.

4. Parameters of the Situation: 1. Travelling at a default $70 \mathrm{mph}, 2$. Other Vehicles and hazards on the road.

\section{Alerts/Sounds for:}

(a) Indication of speed change and why (weather, accident/hazard, traffic density/flow).

(b) Lane changing and why (accident/hazard, maintain speed),

(c) Continuous state of the car- why - normal environment/status.

(d) Manoeuvring off the motorway environment - why handing the driving over to the user.

This scenario is now broken down and entered into the cue sheet. Figure 5 illustrates how this has been done.

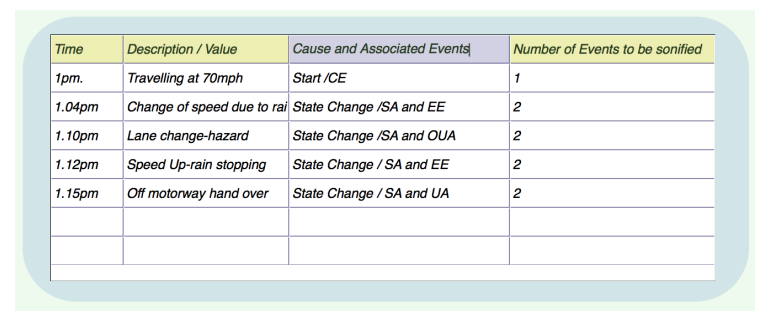

Filled out cuesheet

Figure 5: A filled out cue sheet for the autonomous driving scenario. Each requirement is marked out from top to bottom. The number of events that can be used to represent these requirements of the interface are indicated in column 4. Column 2 represents a place where qualitative and quantitative information can be documented.

\subsubsection{Stage 2: Conceptual Design/Arranging}

At this stage in the design a series of sound suggestions are presented based on the criteria listed above. The designer has the option to audition different sounds and 'upload' them to the events 
mapped out on the timeline. The sounds are based around the following basic mapping principles, however, it should be noted that it is clear that they are suggestions and the designer can of course use any sound they feel suitable. In addition to the list below, it is a feature of the database of sounds that the sounds will harmonically relate to one another. For example, the drone will have a pitch and the motifs will relate harmonically to that pitch. As mentioned, it is up to the designer to make this decision, but one importan feature of SoundTrAD is that the sounds are categorised with that available option.

- User Actions /other user actions: Foley sounds, everyday sounds.

- System actions: Foley sounds, everyday sounds, motifs (melodic and rhythmic variations.). Short sounds (beeps, dings, chimes, and percussive hits) all with the ability to be pitched.

- Environmental events: natural, everyday sounds ranging from organic sounds to industrial.

- Continuous events: drones, soundscapes and ambient-based sounds, ranging from organic sounds to industrial. All with a pitch or ability to be pitched.

\subsubsection{Stage 3: Mapping and Auditioning}

Once the user/designer has uploaded the sounds to the timeline (see Figure 6) they can play the events on the timeline and listen to their selections. Specifically, they can listen to how different events may sound together and subsequently pay attention to aesthetics and potential masking of information. For example, in the driving scenario we know that event 2 and 3 have to represent the fact that the car changes state by slowing down (a system action) due to the rain (an environmental action/event). We are essentially needing to sonify two pieces of information - the slowing down of the car and the start of the rain. The events are placed on the timeline in such a way that the designer can hear these two bits of information that are needed for this requirement simultaneously and subsequently make a decision as to whether the sounds communicate whatever it is they need to be communicating. It may be, for example, the designer wants the sounds to play shortly one after another or overlap. In this case they can re-arrange the events to audition this idea. Another important feature of the timeline is the ability to repeat events. For example, the rain event is represented on line 2 of the timeline and occurs twice during the scenario. However, it occurs alongside different events (the first being the slowing down of the car and the second being the speeding up of the car). As a result, the 'rain sound' can be used twice but may in fact sound very different when played with the 'slowing down sound' and 'speeding up sound', respectively. Figure 6 demonstrates this.

\subsubsection{Stage 4: Usability Evaluation/Auditioning}

The events can be added, auditioned, moved, lengthened, shortened and removed. At any time during the design process the designer and or the end user, or ideally both together, can revisit the scenario and the cue sheet to re-examine their initial design ideas. Importantly, SoundTrAD caters for different use-cases within a given scenario. For example, the events can change order given a different order of user interaction and subsequently different sound

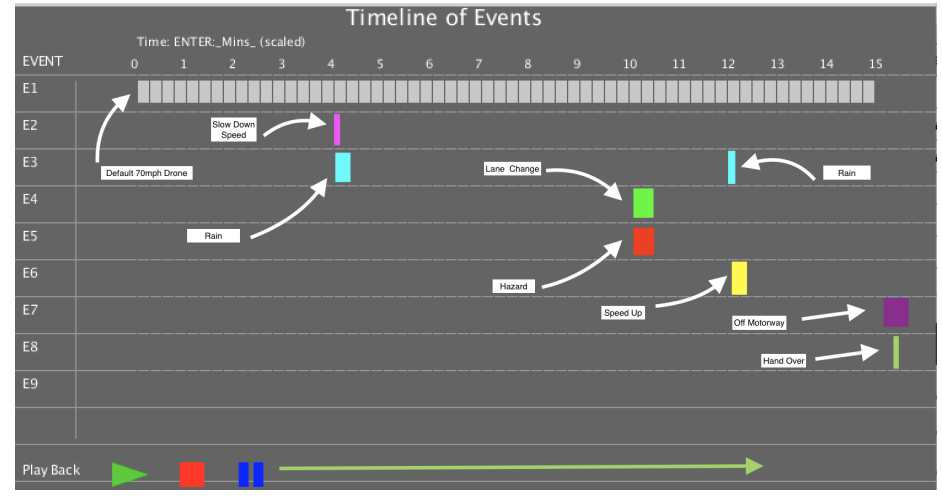

Timeline

Figure 6: The Timeline for the autonomous driving scenario. Events can be moved about, lengthened and shortened. More lines can be added.

combinations can be tested given different possible combinations of events.

\section{DISCUSSION AND FUTURE WORK}

This paper has presented SoundTrAD, a method and tool for prototyping ADs, and applied it to a hypothetical driving scenario. We have argued, based on evidence from a previous study, that SoundTrAD can cater for a wide range of scenarios. In this paper we have applied it to a specific driving scenario to demonstrate its application.

SoundTrAD supports the designer in breaking down a given scenario in to events and systematically mapping them out in order to reconfigure the scenario. As a result, the auditory display can a) cater for different use-cases and b) cater for the application and auditioning of audio and potential issues with masking and aesthetics.

In this example we have taken a limited scenario to demonstrate how a novice AD designer can build an auditory interface that supports an end-user when responding to an autonomous device.

This paper concludes by proposing that this application of SoundTrAD, to a hypothetical driving scenario, can be expanded in order that the driver (end-user) features in the decision making process. Specifically, that the end-user can make decisions around how the $A D$ is incorporated into their interaction with the vehicle. We feel that there are benefits to giving the driver choices over sounds and bespoke personalised alerts. The sound designer could set parameters for the end-user, for example, to introduce change so that the end-user does not become desensitized to the alerts. It could be suggested that giving the end-user a perception of control over the audio in the car will add to their overall sense of confidence, familiarity and trust with the autonomous device. An exciting area of research that we feel SoundTrAD could be applied to.

\section{ACKNOWLEDGMENT}

The majority of research in this paper was funded by the EPSRC, digital economy Media Arts Technology Doctoral Training Cen- 
tre at Queen Mary University of London. The later stages of the work are supported by CHERISH-DE, an EPSRC next stage digital economy research Hub at Swansea University.

\section{REFERENCES}

[1] G. Fauconnier and M. Turner, "Conceptual blending, form and meaning," Recherches en communication, vol. 19, no. 19, pp. 57-86, 2003.

[2] D. MacDonald and T. Stockman, "The development of a method for designing auditory display based on soundtrack composition," in Proc. of ICAD 2013, Meeting of the International Conference on Auditory Display (ICAD), 2013.

[3] D. MacDonald and Stockman, "Lowering the barriers to the creation of interactive auditory displays: an experimental investigation," in Proc. of ISon (Interactive Sonification Workshop), 2016.

[4] E. Brazil and M. Fernström, "Subjective Experience Methods for Early Conceptual Design of Auditory Displays," in Proceedings of the 15th International Conference on Auditory Display (ICAD), 2009, pp. 1-8.

[5] T. Hermann, A. Hunt, and J. G. Neuhoff, "Chapter 1 Introduction," in The Sonification Handbook, T. Hermann, A. Hunt, and J. G. Neuhoff, Eds. Berlin: Logos Publishing House, Berlin, Germany., 2011, ch. Chapter 1, pp. 1-6.

[6] C. Frauenberger, T. Stockman, and M.-L. Bourguet, "A survey on common practice in designing audio in the user interface," in Proceedings of the 21st British HCI Group Annual Conference on People and Computers: HCI... but not as we know it, vol. Volume 1. British Computer Society., 2007, pp. 187-194.

[7] C. Frauenberger and T. Stockman, "Auditory display designan investigation of a design pattern approach," International Journal of Human-Computer Studies, vol. 67, no. 11, pp. 907-922, November 2009.

[8] E. Brazil, "A review of methods and frameworks for sonic interaction design: Exploring existing approaches." Brazil, E. (2010). A review of methods and frameworks for sonic interaction design: Exploring existing approaches. In Auditory Display (pp. 41-67). Springer Berlin Heidelberg., 2009, pp. 41-67.

[9] A. Pirhonen, E. Murphy, G. McAllister, and W. Yu, "Nonspeech sounds as elements of a use scenario : A semiotic perspective," in Proceedings of the 12th International Conference on Auditory Display (ICAD), 2006, pp. 134-140.

[10] S. Barrass and C. Frauenberger, "A communal map of design in auditory display," in Proceedings of the 15th International Conference on Auditory Display, 2009, pp. 1-10.

[11] S. Brewster, P. Wright, and A. Edwards, "The application of a method for integrating non-speech audio into humancomputer interfaces," University of York, Department of Computer Science, 1995., Tech. Rep., 1995.

[12] P. Vickers and B. Hogg, "Sonification Abstraite / Sonification Concrete : An 'Æsthetic Perspectice Space' for classifying Auditory Displays in the Ars Musica Domain," Proceedings of the 12th International Conference on Auditory Display 2006, pp. 210-216, 2006.
[13] A. Dix and S. Brewster, "Causing trouble with buttons," in Ancillery Proceedings of HCI'94, D. England, Ed., 1994.

[14] J. Carroll, Making use: scenario-based design of humancomputer interactions. Cambridge, Massachusetts. London, England: The MIT Press, 2000.

[15] D. Benyon and C. Macaulay, "Scenarios and the hci-se design problem," Interacting with Computers, vol. 14, pp. 397405, 2002.

[16] C. Peres, V. Best, D. Brock, B. Shinn-Cunningham, C. Frauenberger, T. Hermann, G. Neuhoff, L. Nickerson, and T. Stockman, "Audio Interfaces," in HCI Beyond the GUI. Design for Haptic, Speech, Olfactory and Other Nontraditional Interfaces, Kortum, P, Ed. Morgan Kaufman Publishers, 2008, ch. 5 Auditory Interfaces.

[17] D. Benyon, Designing Interactive Systems: A Comprehensive Guide to HCI and Interaction Design, 2nd ed. Pearson Education Ltd., 2010.

[18] M. Chion, Audio Vision. Sound on Screen, C. Gorbman, Ed. Columbia University Press, 1994.

[19] D. Sonnenschein, Sound Design: The Expressive Power of Music, Voice and Sound Effects in Cinema. Michael Wiese Productions, 2001.

[20] R. Beauchamp, Designing Sound for Animation. Focal Press, 2005

[21] T. Holman, Sound for Film and Television, 3rd ed. Elsevier/Focal Press, 2010

[22] D. Ventura, Film Music in Focus, 2nd ed. Rhinegold Publishing, 2010

[23] K. Kalinak, Film Music: A Very Short Introduction. Oxford University Press, Inc, 2010.

[24] S. Lipscomb and D. Tolchinsky, "The role of music communication in cinema," Musical communication, pp. 383-404, 2005.

[25] S. Bødker and C. Nylandsted Klokmose, "Dynamics, multiplicity and conceptual blends in hci," in Proceedings of the 2016 CHI Conference on Human Factors in Computing Systems. ACM, 2016, pp. 2538-2548.

[26] T. Hildebrandt and S. Rinderle-Ma, "Toward a sonification concept for business process monitoring," in International Conference on Auditory Display, 2013, pp. 323-330. 